\title{
Relaciones padres-médicos en oncología: un enfoque cualitativo
}

\section{Parent-Doctor relations in oncology: a qualitative approach}

\author{
C. Grau', M.C. Espada ${ }^{2}$, M.C. Fortes ${ }^{3}$
}

\section{RESUMEN}

El objetivo de este artículo es conocer cómo los padres de niños enfermos de cáncer perciben sus relaciones con el personal sanitario, fundamentalmente con los médicos. Se ha utilizado una metodología de investigación cualitativa y grupal. La muestra la constituyen catorce madres/padres cuyos hijos hace más de dos años que han contraído la enfermedad.

Los padres desean información inteligible, detallada y suficiente. La palabra cáncer tiene un gran estigma social, por lo que se intenta evitar cuando se da información a los padres y niños. La comunicación entre los médicos y padres puede tener momentos de tensión en el diagnóstico, recidivas, etc. Los padres confían en la profesionalidad de los médicos y desean que tengan, además de una buena competencia profesional, cualidades humanas. La elaboración de los informes por los médicos es la tarea más criticada por los padres.

Palabras clave. Oncología. Relación médico-familia. Comunicación. Investigación cualitativa.

\begin{abstract}
We want to learn how parents of children with cancer perceive their relationship with hospital staff, especially with doctors. We used group-based qualitative methodology. The sample is composed of 14 mothers/ fathers whose children contracted the disease more than two years previously.

All parents want information that is both intelligible and detailed. The word cancer has a strong social stigma and is avoided when giving information to parents and to children. Communication between doctors and parents can lead to situations of tension during diagnosis and relapses. Parents trust the professionalism of doctors. Parents also want doctors to be competent and to have human qualities. The preparation of reports by physicians is the task most criticized by parents.
\end{abstract}

Key words. Oncology. Physician-family relationships Communication. Qualitative methodology.
1. Departamento de Didáctica y Organización Escolar. Escuela Universitaria de Magisterio "Ausias March". Universidad de Valencia.

2. Departamento de Psicología Evolutiva y de la Educación. Facultad de Psicología. Universidad de Valencia.

3. Departamento de Psicología Evolutiva y de la Educación. Escuela Universitaria de Magisterio "Ausias March». Universidad de Valencia.

\section{Correspondencia}

Claudia Grau Rubio

Apdo. de correos 22045

46071 Valencia

E-mail: Claudia.Grau@uv.es

Recepción: 1 de junio de 2010

Aceptación provisional: 20 de septiembre de 2010

Aceptación definitiva: 21 de septiembre de 2010 


\section{INTRODUCCIÓN}

El diagnóstico de un cáncer pediátrico es el inicio de un largo camino en las relaciones entre los médicos y los padres de niños enfermos de cáncer, que son decisivas para una adecuada adaptación de los padres y de los niños a las exigencias de la enfermedad. Existen pocos trabajos que aborden las relaciones de los médicos/padres desde la óptica de los padres.

La relación médico/niño/padres es fundamental para el tratamiento oncológico y, cuando no es satisfactoria, suele generar conflictos, dificultades para mantener la adherencia terapéutica, abandono, o exceso terapéutico ${ }^{1}$.

Los médicos son la principal fuente de información de los padres: les comunican el diagnóstico, los resultados de las pruebas, los tratamientos a los que van a ser sometido su hijo, las secuelas de los tratamientos y de la enfermedad, las posibles recidivas o la inmediatez de la muerte del niño. Los psicólogos pueden ayudar a los médicos en los procesos de información a los padres y niños enfermos.

También pueden informarles y aconsejarles acerca de cómo convivir con la enfermedad, cómo continuar con una vida familiar, social y laboral satisfactoria, de qué recursos sanitarios y asistenciales dispone y cómo participar activamente en el tratamiento ${ }^{2}$. Deben resolver las dudas que surgen y dar consejos sobre los cuidados que el niño precisa, sobre cómo normalizar la vida del niño y sobre cómo deben comportarse para que este objetivo sea cumplido. La relación no es únicamente instrumental, sino también emocional ${ }^{3}$.

La participación de los padres en el tratamiento permite la reducción de los tiempos de hospitalización del niño, favorece la hospitalización domiciliaria y, en última instancia, la mejora de la calidad de vida del niño. Esto implica desarrollar procesos educativos protocolizados, adecuados a la enfermedad y a la capacidad de cada familia, e impartidos por los profesionales que se encargan de la atención integral del niño. Su objetivo fundamental es que los padres puedan autoayudarse y asumir responsabilidades $^{4}$. En esta tarea es fundamental la ayuda del personal de enfermería.

La comunicación entre los médicos y padres va más allá de la mera transmisión de datos técnicos y pronósticos del proceso terapéutico, depende de la relación que se establece con el niño y con su familia y de su colaboración en el tratamiento. Siempre debe ser "a la medida" teniendo en cuenta circunstancias y edad ${ }^{5}$. El médico debe ser capaz de crear un ambiente de confianza y seguridad en el niño y en su familia, de escucharlos y de favorecer la expresión de sentimientos y de emociones y la toma de decisiones de forma comparti$\mathrm{da}^{6}$. La vivencia de la enfermedad del niño y de su familia es distinta a la del médico y, a su vez, cada niño y cada familia viven el sufrimiento de forma singular?

La comunicación entre padres y médicos puede tener dificultades cuando se producen recidivas y/o muerte del niño. También puede verse afectada cuando los padres tienen un nivel socio-cultural bajo ${ }^{8}$, son emigrantes y de culturas diferentes ${ }^{3}$ y presentan alteraciones psicológicas.

Por último, los médicos desarrollan una función administrativa, no sólo en la cumplimentación de las historias clínicas ${ }^{9,10}$ y consentimientos informados para el hospital $^{11,12}$, sino también en la elaboración de informes y certificados que les exigen a los padres en otros servicios: sanitarios (médico de cabecera), educativos (informes para los maestros y escuela) y sociales (ayudas, certificados de minusvalía, etc.). Esta actividad es de gran relevancia para la mejora de la calidad de vida de los niños enfermos de cáncer y sus familias y, a su vez, constituye una sobrecarga para los equipos médicos.

El objetivo del presente estudio es describir cómo perciben los padres de niños enfermos de cáncer sus relaciones con el personal sanitario, fundamentalmente con los médicos. Este objetivo forma parte de un trabajo de investigación que pretende conocer, además del objetivo señalado, las estrategias de afrontamiento que utilizan los padres y cómo perciben los cambios 
que se producen en la estructura y dinámica familiar. Para conseguir estos objetivos hemos utilizado una metodología cualitativa de carácter grupal.

\section{MÉTODO}

Se ha utilizado una metodología cualitativa y grupal. La metodología grupal (sesiones de grupos de autoayuda) ha sido utilizada en otros estudios relacionados con padres de niños y adolescentes con cáncer en diferentes fases de la enfermedad ${ }^{13-17}$.

Frente a los objetivos perseguidos en la mayoría de dichos estudios (intervención psicológica de los padres), este trabajo de investigación pretende conocer las estrategias de afrontamiento que han utilizado los padres y los cambios que se han producido en la dinámica familiar, también la forma en que perciben las relaciones con los médicos.

No se ha utilizado el formato de grupo de autoayuda sino el de grupo de discusión, ya que nuestro objetivo no es terapéutico sino recabar información.

El grupo de discusión consiste en la reunión de un pequeño número de personas para obtener información en un ambiente agradable con un formato de conversación que estimula el debate y la confrontación.

Se trata de reflejar la comprensión de la realidad (relaciones de los padres con el personal sanitario...) a través de la participación de diferentes actores (padres de niños con cáncer). Se realiza una entrevista grupal abierta entre personas y en su discurso se muestran relaciones relevantes para el objeto de estudio planteado. El material obtenido es cualitativo y es objeto de análisis de contenido y del discurso.

El grupo lo forman diez madres y cuatro padres cuyos hijos habían sido diagnosticado de cáncer hacía más de dos años y habían sido tratados en los hospitales de Valencia: el Clínico Universitario y La Fe. En la muestra participaron padres que han pasado por algunas de las fases de la enfermedad (diagnóstico, tratamiento, recidivas y muerte).

Dada la dificultad de acceder a este colectivo, el procedimiento de muestreo utilizado ha sido el probabilístico por conveniencia, es decir, una muestra incidental construida a partir de los padres a los que se ha tenido acceso, gracias a la colaboración de ASPANION (Asociación de padres de niños oncológicos de la Comunidad Valenciana).

La mayoría de los participantes son madres (diez); todos son miembros de ASPANION y han recibido sus servicios de apoyo psicológico si lo han solicitado. Pocos han participado en sesiones grupales. El grupo se formó exclusivamente para este trabajo de investigación y contó con el consentimiento de todos los padres, que participaron oportunamente.

Se realizaron seis sesiones grupales (más una sesión de presentación y otra de despedida) de hora y media de duración cada una de ellas y estuvieron dirigidas por la psicóloga $\mathrm{M}^{\mathrm{a}}$ Carmen Espada, en la sede de ASPANION de Valencia.

La sesión de presentación fue a cargo de un profesional de la asociación para explicarles el proyecto y que decidieran quiénes estaban dispuestos a participar. En la primera sesión se expusieron los objetivos de la investigación y se les pidió su consentimiento y la autorización para grabar todas las intervenciones; asimismo, se lanzaron algunas preguntas motivadoras para iniciar el debate.

Las cinco sesiones siguientes versaron sobre: los problemas a los que se tuvieron que enfrentarse, las estrategias utilizadas para solucionarlos, los cambios producidos en su vida familiar y las relaciones con el personal sanitario.

En la sesión de despedida se recordó que los datos recogidos eran para una investigación y que contábamos con su consentimiento. Se les dio las gracias por su participación y se hizo un resumen de los temas tratados en las sesiones grupales.

Aunque hubo un diseño inicial de las sesiones, atendiendo a la revisión bibliográfica, éstas se fueron adaptando a la dinámica de cada una, respetando la espontaneidad de los participantes. Las sesiones fueron grabadas en audio y reproducidas literalmente por escrito. 
Finalmente, se realizó un análisis del contenido de las grabaciones, organizándolo en categorías, subcategorías y palabras clave. Las categorías fueron las siguientes:

- Estrategias de afrontamiento: resolución de problemas, regulación afectiva, búsqueda de información y apoyo social, reevaluación o reestructuración cognitiva, distracción, rumiación, huida/evitación y aislamiento social. Para establecer las categorías se siguió el meta-análisis de estrategias de afrontamiento de Campos y col ${ }^{18}$.

- Cambios en la dinámica familiar: relaciones de pareja, con el niño enfermo, con los otros hijos y con la familia extensa, dificultades en la vuelta a casa después de la hospitalización.

- Relaciones con los médicos: información a los padres y a los hijos, confianza en la profesionalidad de los médicos y valoración del trato recibido, quejas, consejos de los médicos a los padres, situaciones de tensión, e informes.

En cada categoría y subcategoría se seleccionaron algunas frases que sintetizan y representan las diferentes opiniones de los padres.

\section{RESULTADOS}

\section{Información a los padres}

A los padres no les gusta cómo los médicos les dan la información, el lenguaje que utilizan, y prefieren que les den solamente la información necesaria:

- "Los médicos no nos miran como padres y madres; nos miran y nos hablan como médicos".

- «Nosotros queríamos saber lo justo, no queríamos que los médicos nos dieran más información que la necesaria".

- "Cuando empecé a investigar y me enteré de la enfermedad y las secuelas de la enfermedad que iba a dejar en mi hijo, me di cuenta que los médicos no dicen toda la verdad, porque la verdad es muy dura».

\section{Necesidad de información sobre los procesos de la enfermedad}

Constatamos la dificultad de los médicos en dar una información adecuada a los padres, no sólo en el diagnóstico sino a lo largo del todo el proceso de la enfermedad. Observamos cómo algunos padres no se enteraron del diagnóstico de la enfermedad de su hijo:

- "Yo tengo una queja gorda, gorda, que la dijimos después. Juani estuvo un mes ingresada y a nosotros nadie nos dijo que tenía cáncer. Nos decía que era un bulto que le había salido, pero yo ¿por qué tengo que saber que un tumor es lo mismo que un cáncer? Tardaron un mes en decirlo. Ellos decían que nos lo habían dicho".

Todos los informantes exigen y prefieren que los médicos les den una información exhaustiva sobre los cambios que se producen en el hijo como consecuencia de las intervenciones quirúrgicas y de los tratamientos, y sobre las secuelas que pueden producir discapacidad, etc. La información sirve para controlar el impacto que estos efectos negativos produce en los padres. El personal de enfermería en algunas ocasiones suple la falta de información de los médicos:

- «Si no te lo dicen te asustas. Si te lo dicen: mira, te puede pasar esto y esto, no te asustas tanto; pero que te lo digan".

- «Los médicos te tendrían que preparar para decirte cómo van a salir los niños después de la operación, porque cuando yo vi a mi hijo después de la operación, en la habitación con máquinas por todas partes, sondado por aquí, sondado por allí (me asusté). Ellos lo que te tendrían que decir es: 'si esta máquina se para, no pasa nada; si el gotero se termina, esta máquina te avisa'». 
- «Yo sabía que la habían operado de la cabeza, pero lo que no esperaba es que la pusiera de pie y se me cayera. Me di un susto cuando la levanto de la silla de ruedas; me giro y me la veo en el suelo y le pregunto: ‘¿pero qué haces ahí?' y me dice: 'que me he caído'. La vuelvo a poner de pie y se me vuelve a caer otra vez. Lo que yo pensé es que esta niña se me ha quedado inválida y nadie me ha dicho nada. Luego le pregunté a la enfermera: '¿oye, que la niña se me ha quedado inválida?' y ella me dijo que en la operación de la cabeza tenía que pasar un tiempo, ya que las piernas se resentían. Pero, claro, a mí me lo dicen y no me pegan esos sustos".

Normalmente, los médicos informan a los padres sobre la inmediatez de la muerte del niño:

- "Los médicos saben cuándo la muerte está cercana y te avisan en ese momento, por si quieres estar con el niño en ese momento. Y los padres deciden si se llevan los niños a intensivos para que se mueran allí o los dejan en la habitación (del hospital) para que fallezcan allí».

Así pues, los padres desean información sobre todos los aspectos de la enfermedad: tratamientos, secuelas, etc. Consideran que la información debe darse de una manera inteligible y detallada en la explicación de los procesos y secuelas y debe ser suficiente, aunque no demasiada.

\section{Información a los hijos}

\section{Estigma de la palabra cáncer}

La palabra cáncer tiene un gran estigma social, por lo que intenta evitar sustituyéndola por la palabra tumor. En los niños más mayores, los médicos utilizan la palabra cáncer, si éstos lo piden. En los niños más pequeños, se evita utilizar la palabra cáncer y, normalmente, son los padres los encargados de dar la información a sus hijos. La misma tónica se utiliza en todas las informaciones relativas a la enfermedad: en los más mayores, suele ser el médico en colaboración con los psicólogos y, en los más pequeños, se suele delegar en los padres:

- «En el momento que los médicos supieron que era cáncer, se lo dijeron a ella. Le dijeron que tenía un tumor maligno y luego, de ahí, nos subieron a la psicóloga. Ella le preguntó ‘¿es un cáncer?' y la psicóloga le dijo que sí. Ellos nos dijeron que con la edad que tenía era mejor que se lo dijéramos, porque todo lo que le iba a pasar (que se le cayera el pelo) ella no lo iba a comprender e iba a pensar que estaba aún peor de lo que realmente estaba y eso a ella la iba a hundir".

- «La palabra cáncer asusta mucho. A Lucía le dijeron los médicos en todo momento la palabra tumor y le explicaron 'se te va a caer el pelo y esto y lo otro', pero siempre con la palabra tumor. Y luego nos hicieron pasar con la psicóloga del hospital La Fe y entonces mi hija le preguntó: ‘tengo cáncer?' y la psicóloga dijo: 'sí'. Se te cae el mundo encima y no reaccionas hasta que pasan unos meses".

- "A nosotros nos dijeron que debíamos decírselo cuanto antes. Entonces yo pregunté: ‘¿pero cómo le digo esto al niño?', y me dijeron que como la palabra cáncer él no la entendía que le explicará que aquello era como un constipado grande y que en el hospital le iban a curar y que, aunque le iban a hacer daño, él tenía que estar ahí y así se lo dijimos».

- «Desde el primer día que yo lo tuve claro le expliqué a mi hija con 5 años lo que tenía. Se lo expliqué con la ayuda de los folletos que ASPANION daba. Luego cuando íbamos periódicamente al hospital (para hacer revisiones, análisis, tratamiento, etc.), yo siempre le decía la verdad. Ella confiaba en mí y ella me preguntaba: ‘ ¿pero me van a pinchar?' y yo le decía: 'no, Juani, ¿yo te he mentido alguna vez?', no, me decía ella». 


\section{Información de las secuelas a los hijos}

También es complicado para los padres y profesionales informar a los niños de secuelas graves, que pueden provocar discapacidad:

- "Cuando yo le tuve que explicar las posibles secuelas de la operación a mi hija de 3 años, se me hizo una montaña. Me dijeron que en la operación se podía quedar muda, ciega, sorda, o sea, cualquier cosa podía pasar. Y le dije a la niña: 'te van a quitar el bichito que tienes en la cabeza que te está haciendo pupa y para eso te van a llevar a una habitación, donde hay una ventanita pequeñita, tú no me vas a ver, pero yo sí que te veo por la ventanita, tú no me oyes pero yo sí que te oigo. Lo que pasa es que cuando salgas de ahí igual no vas a poder hablar, pero tú no te preocupes que ya hablarás y a lo mejor tampoco vas a poder oír; pero tú no te preocupes que ya oirás, y si pasa que no me ves a mí, ni nada, tú tampoco te preocupes', y se quedó tranquila".

Los niños más mayores exigen información sobre todos los procesos y secuelas de la enfermedad, incluso cuando pueden derivar en discapacidad. Los psicólogos son de ayuda en estos procesos:

- «Ella dijo que se lo contaran todo y entonces le dijeron (los médicos) que le podían cortar la pierna».

- "La psicóloga de La Fe (hospital) hacía a Luisa simulacro de punciones lumbares, para que se preparara. Ella quería ver todo lo que le hacían $\mathrm{y}$, claro, las punciones lumbares difícilmente las podía ver. Total que, al final, había que hacerle simulacro de punciones paso a paso y le decía: 'ahora te voy a pinchar, ahora te estoy metiendo el líquido, ¿lo notas?, está fresquito, etc.'. Y esto fue una idea del psicólogo y gracias a él, qué preparó a Luisa en los simulacros, a Luisa le hacían las punciones sin el medicamento. Se ponía un poco ner- viosa, lógicamente, pero ya no era lo del principio".

\section{Confianza en la profesionalidad y valoración del trato recibido}

El trato con los médicos en general es bien valorado; además, confían en su actuación cuando los hijos se encuentran mal:

- "Yo le pongo un diez a la doctora de Luisa en todos los aspectos, como profesional y como persona. Tiene una calidad humana impresionante, pero yo sé que esa persona es muy joven y se 'rayará', se 'rayará' seguro".

- «En los médicos no he notado la falta de sensibilidad. A los médicos los he visto un poco serios, no se relacionan tanto. Pueden estar más o menos simpáticos, pero hacen su trabajo".

- «Sabes que, cuando vienen los médicos, van a hacer todo lo posible por animarla».

- "Que vengan pronto los médicos y que miren que todo está bien. Cuando tu hijo está mal, como tú no puedes hacer nada, te sientes impotente. Dejas a tu hijo en manos de los médicos, porque crees que es la mejor solución".

Los padres están satisfechos en general con el trato que les dio el personal de enfermería. Incluso señalan cómo después de dejar el servicio se acuerdan y preguntan por su hijo:

- «Las enfermeras se portaron muy bien".

- «Ahora cuando voy a rehabilitación todos me preguntan cuando me ven. Claro, estuve allí tanto tiempo que ahora cuando me ven me preguntan cómo estoy y cómo está el niño".

- «La enfermera le había cogido mucho cariño y recuerdo a la enfermera limpiándolo y llorando a la vez. A ella le afectó mucho" (comportamiento ante un niño en fase terminal). 


\section{Quejas respecto al trato recibido por los médicos y a la competencia profesional de los médicos noveles}

Algunos padres señalan la importancia de que los médicos los traten bien personalmente. También se quejan del trato recibido:

- "Yo creo que los médicos debían de hacer no sólo los estudios sino también trato con la gente».

- "Yo no me esperaba de La Fe este trato. No es lo mismo que vayas a un médico de cabecera para un constipado que vayas a un sitio de oncología, a un sitio que te tienes que hacer unas pruebas que te pueden dar mal resultado por lo que sea, pues esas cosas las tienen que tratar con más delicadeza, pienso yo".

- "Hay pocas personas, de médicos y enfermeras, que a la hora de hablar con la gente sean agradables".

- «A las enfermeras les falta sensibilidad".

- «Tendrían que prepararlas psicológicamente".

Los padres también se quejan de la falta de competencias de los médicos noveles:

- «Los niños también pagan muchas veces la inexperiencia. Yo pienso que los niños, cuando están muy nerviosos, lo debería de hacer un profesional y que las de prácticas lo practiquen en otros pacientes. Lo que no se puede hacer es que pinchen a tu hijo cinco o seis veces, que no le atinen, que le hagan sangre y que luego ya no se la puedan hacer porque lo han 'machacado'. Yo creo que si en la primera vez o en la segunda no lo atinan, como mucho dos veces y no más, debería venir el profesional que en un momento lo hace bien, así ni sufre el niño, ni sufre la mamá, ni sufren los médicos. Por ejemplo, la doctora, le pinchó tres veces y le dijo: 'no te pincho una vez más porque es demasiado'. Salió llorando la doctora, vino otra y se lo hizo".
Así pues, los padres confían en la profesionalidad de los médicos cuando hay problemas. Desean que los médicos y el personal de enfermería tengan, además de una buena competencia profesional, cualidades humanas para el contacto emocional con los padres. Se quejan de los profesionales que no tienen estas cualidades. Están de acuerdo en que los médicos noveles adquieran competencias profesionales siempre que no sea a costa del sufrimiento de sus hijos.

\section{Consejos de los médicos a los padres}

Los consejos que reciben los padres de los médicos abarcan una gran gama de comportamientos, referentes a la normalización de la vida del niño, a la adquisición de competencias para el manejo de la enfermedad y consejos prácticos para la vuelta a casa:

- «El médico me dijo: 'tu hija te debe ver siempre bien, si estás cansada que te vea siempre con el mejor aspecto, maquíllate'».

- "Y cuando luego fuimos a La Fe (al hospital $\mathrm{La} \mathrm{Fe}$ ) y allí me dijeron que Luisa siempre me tenía que ver bien, pues ésa fue la política que seguí siempre".

- «Los médicos aconsejaban que en casa no hubiera mucha gente, porque los niños estaban bajitos de defensas, así que yo lo primero que hice es decir que vinieran padres, hermanos y nadie más».

- «Es lógico que los médicos quieran que lo hagan (las curas y cuidados) las madres, así los niños lo ven mejor".

\section{Situaciones de tensión en las relaciones con los médicos}

Observamos en muchos casos la dificultad en el diagnóstico inicial de la enfermedad. Se quejan de la tardanza del diagnóstico:

- "Yo fui dos semanas al Hospital Provincial y me decían que mi hijo no 
tenía nada. Cuando lo detectaron, el médico me dijo que se lo habían diagnosticado en una fase avanzada".

Otro momento de tensión es cuando se produce una recidiva en algún niño:

- "Al año diagnosticaron a Jorge de un tumor en la cabeza y cuando tenía tres años diagnosticaron una metástasis en la médula espinal. Cuando Jorge recayó, se dieron cuenta que el cáncer de la cabeza que tenía se había reproducido en metástasis en la espalda, en la médula espinal. Yo les pregunté a los médicos qué por qué no le habían mirado antes todo el sistema nervioso si la médula espinal también pertenece al sistema nervioso central y mi hijo tenía un tumor en la cabeza".

\section{Informes}

Respecto a los informes, los padres se quejan de que se hacen de manera provisional y de que no contienen información exhaustiva de la enfermedad del hijo. Los informes son necesarios para solicitar los certificados de minusvalía o son requeridos por otros servicios hospitalarios donde atienden al niño. En estos comentarios podemos observar también la falta de coordinación y comunicación entre diferentes servicios de un mismo hospital:

- «Me faltan muchos informes porque, con eso de que te quieres ir rápido a casa, te dan un informe provisional que no pone nada, todo en siglas. Pero los informes en donde realmente cuentan toda la historia no están".

- «El último informe de alta tardaron un año en dármelo y porque lo necesitaba para arreglar el tema de la minusvalía".

- "Yo el último informe de Jorge, aún no lo tengo. Me lo pidieron en rehabilitación y yo lo pedí al médico y, al final, en rehabilitación han dejado de pedírmelo porque han visto que nadie me lo da".

\section{DISCUSIÓN}

La metodología cualitativa nos ha permitido acercarnos al punto de vista de los padres sobre sus relaciones con los médi$\cos$. Hemos utilizado una metodología grupal, que, junto con las entrevistas semiestructuras, son las técnicas más utilizadas en el ámbito de la salud ${ }^{2,8,19}$.

La técnica de muestreo "probabilístico por conveniencia" utilizada es uno de los aspectos más débiles de este trabajo de investigación. La dificultad de acceder a este colectivo nos ha impedido establecer diferencias, entre padres y madres, y entre padres de niños de reciente diagnóstico, en periodo de tratamiento, recidivas y muerte. Normalmente estas limitaciones suelen ser habituales en investigadores que no trabajan en los servicios hospitalarios y, por lo tanto, recurren a las asociaciones.

Las relaciones y comunicación médicofamilia es un tema que preocupa a los médicos y se recoge en los libros de oncología pediátrica $^{20}$. Sin embargo, existen pocos trabajos desde la perspectiva de los padres. Consideramos que el punto de vista de éstos puede ser de gran utilidad para el personal sanitario en su práctica profesional.

Los resultados de nuestro trabajo concuerdan con los de otros trabajos en cuanto que los padres quieren información, confían en la profesionalidad de los médicos y desean que los médicos y personal de enfermería tengan, además de una buena competencia profesional, cualidades humanas para el contacto emocional con los padres; sin embargo, también hay problemas de comunicación con los médicos. Hay que mejorar la forma de dar la información en las diferentes fases de la enfermedad y de atender las cuestiones administrativas referentes a la elaboración de los informes que les exigen a los padres en otros servicios.

Consideramos que este trabajo constituye una primera aproximación al estudio de cómo perciben los padres su relación con los médicos, a pesar de su dificultad: objetivos de investigación amplios y una muestra reducida de participantes. 
Para trabajos futuros se pueden utilizar entrevistas semiestructuradas y grupos de discusión específicos. A partir de los resultados obtenidos en las entrevistas y grupos se podría elaborar un cuestionario que permitiera conocer la opinión de un mayor número de padres.

Creemos que los resultados de estas investigaciones pueden ser de gran utilidad en el diseño de las competencias profesionales en los programas de formación de los médicos y en la mejora de su práctica profesional.

Por último, las relaciones padres-médicos pueden ser objeto de investigación en el ámbito de la organización del sistema sanitario y las vivencias de los padres (como usuarios del sistema) ser utilizadas para mejorar la asistencia sanitaria.

\section{BIBLIOGRAFÍA}

1. Medina-Sansón A, Adame-Vivanco MJ, SchererIBARRA A. Obstinación Terapéutica. Bol Med Hosp Infant Mex 2006; 63: 287-290.

2. Gatte L, Cantero P, González Lamuño D, García FuEnTES M. Necesidades de los pacientes pediátricos con enfermedades raras y de sus familias en Cantabria. Madrid: Real Patronato sobre Discapacidad, 2005.

3. ÁlVAREZ F, Fortin S, Bibeau G. La práctica pediátrica en un medio cultural plural: una experiencia en curso. Arch Argent Pediatr 2008; 106: 236-241.

4. DíAz CA. Educación sanitaria a padres y niños con asma. FMC, Formación Médica Continuada en Atención Primaria 1999; 6: 611-623.

5. Prado C, Azcona I, Rodríguez E, Hernández M (2007). La comunicación en oncología pediátrica desde el punto de vista del cirujano. Psicooncología 2007; 4: 191-202.

6. CRuz M. Un renovado reto en la formación pediátrica: la relación médico-paciente. Educ med 2004; 7: 119-124.
7. Ayarra M, Lizarra S. Malas noticias y apoyo emocional. An Sist Sanit Navar 2001; 24 (Supl. 2): 55-64.

8. Garrafa V, Albuquerque MC. Enfoque biotético de la comunicación en la relación médico paciente en las unidades de terapia intensiva. Acta Bioeth 2001; 7: 355-367.

9. Bertrán JM, Collazo E, Gervás J, GonzálezSalinas P, Gracia D, Júdez J, Rodríguez JJ, Rubi $\mathrm{J}$ y SÁNchez M. Intimidad, confidencialidad y secreto. Madrid: Fundación de Ciencias de la Salud, 2005.

10. Iraburu M. Confidencialidad e intimidad. An Sist Sanit Navar 2006; 29 (Supl. 3): 49-59.

11. MuÑoz G, Rivera JA y RoJAs NE. La bioética en la toma de decisiones médicas en niños hospitalizados. Rev Mex Ped 2004 71: 243-247.

12. Simón P. Diez mitos en torno al consentimiento informado. An Sist Sanit Navar 2006; 29 (Supl. 3): 29-40.

13. Collette R, Collette L. Psychological support to parents with children with cancer. J Child Psychol Psychiatr 2003; 3: 15-25.

14. Dellepiane R, Eandi P, Endrizzi C. Gruppi dei genitori con i bambini con la leucemia. Psicologia e Malattia 2003; 15: 11-21.

15. Popoliko C, Rumino PL. Cerebral cancer: a support to the parents. Adv Ther 2003; 27: 96102.

16. Rumino C, Rumino PL. Helping parents with children with leukaemia. Adv Ther 2002; 24: 55-59.

17. Vaamonde L, Moreno P, Carunchio JM, Gibeluin R, VACCARO M. Bambini con cancro nella fase finale. Psicologia e Malattia 2001; 6: 62-70.

18. Campos M, Iraurqui J, Páez D, Velasco C. Afrontamiento y regulación emocional de hechos estresantes. Un meta análisis de 13 estudios. Bol Psicol 2004; 82: 25-44.

19. GonZÁLEZ P. Experiencias y necesidades percibidas por los niños y adolescentes con cáncer y sus familias. Nure Inv 2005, 16: 1-15.

20. Sierrasesúmaga L, Antillón F, Bernaola E, Patiño A, San Julíán M. Tratado de Oncología Pediátrica. Madrid: Pearson, 2006. 
